\title{
New strategies for cancer management: how can temozolomide carrier modifications improve its delivery?
}

\author{
"Different strategies, including carrier-mediated and receptor- \\ mediated transport, cell-penetrating peptides and cell-mediated \\ delivery, must be critically looked into to enhance the permeability of \\ the blood-brain barrier for improved temozolomide delivery."
}

First draft submitted: 31 January 2017; Accepted for publication: 14 February 2017; Published online: 21 June 2017

Keywords: cancer $\bullet$ glioblastoma $\bullet$ nanoparticles $\bullet$ polymer drug conjugates $\bullet$ targeting - temozolomide

Glioblastoma multiform (GBM) is the most devastating, highly aggressive astrocytic cell neoplasm having a median survival of 12-15 months and a 5-year survival rate of $<3 \%$ [1]. Surgery along with radiation therapy and/or chemotherapy is the standard treatment strategy for primary brain tumors wherein, the survival advantages are only palliative. Despite clinical and technological advances, a cure for GBM remains elusive due to its diffuse infiltrative pattern of growth (hindering complete surgical resection), cytogenetic heterogeneity (limiting the use of pathway-specific targeted agents) and location (need to cross the blood-brain barrier [BBB]). Temozolomide (TMZ) is the first-line chemotherapy for GBM used in conjunction with radiotherapy or as a single agent for maintenance therapy [1]. It is an imidazotetrazine class DNA alkylating agent that methylates guanine and adenine bases of DNA leading to DNA double-strand breaks, cell cycle arrest and eventual cell death [1]. An autophagy induction leading to cell death has also been reported as a putative mechanism of action of TMZ in cancer cells and GBM patients [2]. Looking at the current therapy for GBM, there is still an unmet medical need resulting due to its inefficient delivery of TMZ to the cancer tissue. Only a modest activity is seen for TMZ, particularly in high-grade gliomas, which is further limited by the development of resistance leaving no viable therapeutic option for recurrent glioblastoma [3]. Further, TMZ is an unstable molecule that undergoes rapid hydrolysis and has significant dose-limiting hematological toxicity that prevents dosage increase [1]. Currently, TMZ is given orally or intravenously (TEMODAR ${ }^{\circledR}$ ) at a dose of $75 \mathrm{mg} / \mathrm{m}^{2}$ concomitant with radiotherapy for 49 days followed by $150 \mathrm{mg} / \mathrm{m}^{2}$ (cycle 1) and $200 \mathrm{mg} / \mathrm{m}^{2}$ (cycle 2-6) as a maintenance dose.

Efforts have been made to develop efficient and safe nano-carrier systems for conventional chemotherapeutic drugs that offer various advantages, including passive/active targeting, reduced side effects, increased maximum tolerated doses and overcoming chemoresistance. To avoid systemic side effects of TMZ, targeting to the brain would be of paramount importance. One way to achieve high drug concentrations in the brain is localized delivery directly into the brain tumor by using a mixture of biodegradable polyanhydride polymer (poly[1,3-bis(pcarboxyphenoxy)propane (CPP)]-sebacic acid [SA] copolymer) and TMZ, as this had significantly prolonged the survival time of experimental animals [4]. However, local administration would have limited translation potential due to its invasive nature and

\section{Saurabh Sharma}

Department of Pharmacy, Birla Institute of Technology and Science (BITS)-Pilani, Rajasthan, India

Kishan Italiya

Department of Pharmacy, Birla Institute of Technology and Science (BITS)-Pilani, Rajasthan, India

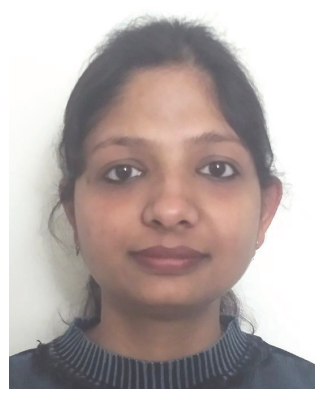

\section{Anupama Mittal}

Department of Pharmacy, Birla Institute of Technology and Science (BITS)-Pilani, Rajasthan, India

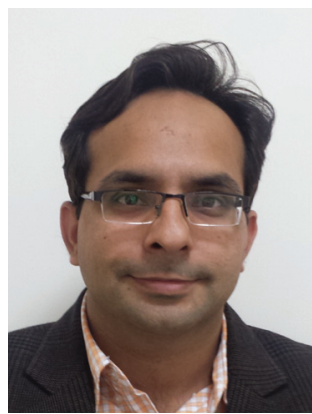

Deepak Chitkara*

Department of Pharmacy, Birla Institute of Technology and Science (BITS)-Pilani, Vidya Vihar Campus, Pilani, Rajasthan 333031 , India

*Author for correspondence:

Tel.: +9101596515835

deepak.chitkara@pilani.bits-pilani.ac.in

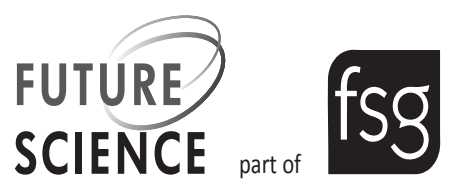


the drug needs to be administered only at the time of surgery thus, a systemic and targeted delivery of TMZ appears to be a more beneficial treatment approach. However after systemic delivery, one of the major hurdles is getting drugs into the brain as they need to cross the BBB. Thus, much research is focused on modifying the carrier properties to enable $\mathrm{BBB}$ penetration in order to target the GBM.

\section{Nanoparticles for TMZ delivery}

For TMZ delivery, nanoparticles and polymer-drug conjugates have demonstrated significant improvement in cell-based assays and preclinical studies. Tian et al. showed that polysorbate 80-coated polybutylcyanoacrylate nanoparticles resulted in better uptake of TMZ by the brain as compared with TMZ solution and non-coated TMZ nanoparticles [5]. To achieve targeted TMZ delivery, Jain et al. have prepared transferrin appended PLGA-PEG nanoparticles of TMZ wherein a $7.5 \% \mathrm{w} / \mathrm{w}$ loading was obtained. Further, an enhanced cellular uptake and cytotoxicity were observed with targeted formulations [6]. Recently, Irani et al. loaded TMZ in chitosan nanoparticles that were subsequently incorporated into polyurethane nanofibres with more than 90\% encapsulation efficiency and sustained TMZ release for 30 days. The surface of the nanofibers was further modified with gold nanoparticles to enhance activity in GBM cells [7]. Chitosan-based nanoparticles were also studied for co-delivering TMZ with other therapeutic molecules including miRNAs. Fan et al. reported sequential release drug delivery system consisting of anionic gold nanoparticles and folic acid decorated chitosan nanogels for co-delivery of miRNA-218 mimic and TMZ for malignant gliomas that showed significantly enhanced intracellular uptake and efficacy due to targeting function [8]. In another study, TMZ was co-delivered with doxorubicin using chitosan-grafted-functionalized polylactic acid nanoparticles of 150-300 nm size [9]. Apart from polymeric nanoparticles, solid lipid nanoparticles (SLNs) have also been used for TMZ delivery that showed improved brain targeting efficiency of TMZ in mice as compared with TMZ solution [10]. Nanostructured lipid carriers (NLCs), the newer generation of SLNs, consisting of solid and liquid lipids were also prepared as they offer better loading efficiency due to the imperfect crystal structure. Recently, Song et al. reported arginine-glycine-aspartic acid peptide (RGD) modified TMZ loaded NLCs that significantly inhibited the growth of U87 MG tumors in mice [11]. NLCs were also fabricated and compared with SLNs for co-delivery of TMZ with vincristine. These nanoparticles showed a size of $117.4 \mathrm{~nm}$ with an encapsulation efficiency of more than $85 \%$ and a nearly complete release of both drugs within $48 \mathrm{~h}$. Further, vincristine and TMZ loaded NLCs showed significantly better cytotoxicity to U87MG cells and glioma inhibition in mice as compared with SLNs [12].

\section{Polymer drug conjugates for TMZ delivery}

Although TMZ delivery using nanoparticles has shown significant improvement but, there still remains formulation related challenges that need to be addressed, particularly TMZ loading and its release from these systems. TMZ is a water soluble molecule $(5.0 \mathrm{mg} / \mathrm{ml})$ and is hence difficult to encapsulate into polymeric or lipidic nanoparticles. In a recent study by Ananta et al., TMZ was delivered using PLGA nanoparticles wherein a loading of $4.4 \% \mathrm{w} / \mathrm{w}$ was obtained, however, $80 \%$ of TMZ was released within $6 \mathrm{~h}$ [13]. The same has been substantiated in a study by Lee and Ooi wherein, the drug loading of $0.2-2 \%$ was achieved in folate conjugated PLGA-PEG nanoparticles [14]. Another approach to efficiently deliver TMZ is by using polymer-drug conjugate that has shown improved TMZ payload, control over its release characteristics and suitable modification with targeting ligands (including peptides and antibodies) promoting accumulation in GBM. Patil et al. conjugated TMZ in its hydrazide form with poly $(\beta-\mathrm{L}-$ malic acid) along with an antitransferrin receptor (TfR) monoclonal antibody for tumor targeting by receptormediated endocytosis and $\mathrm{pH}$-sensitive trileucine for disruption of endosomal membranes [15]. These conjugates showed high TMZ payload (17\% w/w) and achieved active targeting to GBM. Further, the biological half-life of TMZ was significantly enhanced, (about 3-4 times) after conjugation with the polymer [15]. In another study, TMZ was conjugated with chitosan to obtain nanoparticles with the size of $47.8 \mathrm{~nm}$ and TMZ loading of $4.9 \% \mathrm{w} / \mathrm{w}[1]$. The surface of the nanoparticles was modified with targeting peptide, chlorotoxin, which specifically binds to GBM cells. These nanoparticles showed a marked increase in aqueous and serum stability of TMZ as well as improved cellular uptake (a 2-fold increase in U-118 MG, SF767, and GBM6 cells) and improved in vivo blood-brain permeability [1]. Apart from polymeric carriers, TMZ was conjugated with perillyl alcohol that showed efficacy in TMZ-resistant glioma cells and in orthotopic GBM tumor model [16]. The available reports on TMZ delivery clearly indicate the potential of nanocarriers to achieve a better therapeutic outcome, higher target tissue concentration, improved efficacy and lower toxicity in GBM.

\section{Future perspective}

TMZ delivery using nanocarriers has been able to improve its efficacy, however, there is still a long way 
to go to achieve a decent prognosis in GBM. Both nanoparticles and polymer-drug conjugates have been reported to enhance the delivery of TMZ. By modifying the surface and size characteristics of these nanocarriers, efficient delivery of TMZ could be designed to overcome the delivery hurdles of GBM. Carrier modifications are particularly required to cross the $\mathrm{BBB}$ and target GBM cells. Different strategies, including carrier-mediated and receptor-mediated transport, cellpenetrating peptides and cell-mediated delivery, must be critically looked into to enhance the permeability of the BBB for improved TMZ delivery. Combination chemotherapy with pathway-specific targeted drugs could be another avenue to overcome drug resistance, maximize the therapeutic effect and reduce side effects. However, such systems are complex and require intense and exhaustive efforts to design rational combination modalities. Another alternative noninvasive approach

\section{References}

1 Fang C, Wang K, Stephen ZR et al. Temozolomide nanoparticles for targeted glioblastoma therapy. ACS Appl. Mater. Interfaces 7, 6674-6682 (2015).

2 Catalano M, D'Alessandro G, Lepore F et al. Autophagy induction impairs migration and invasion by reversing EMT in glioblastoma cells. Mol. Oncol. 9, 1612-1625 (2015).

3 Chong DQ, Toh XY, Ho IA et al. Combined treatment of Nimotuzumab and rapamycin is effective against temozolomide-resistant human gliomas regardless of the EGFR mutation status. BMC Cancer 15, 255 (2015).

4 Brem S, Tyler B, Li K et al. Local delivery of temozolomide by biodegradable polymers is superior to oral administration in a rodent glioma model. Cancer Chemother. Pharmacol. 60, 643-650 (2007).

5 Tian XH, Lin XN, Wei $\mathrm{F}$ et al. Enhanced brain targeting of temozolomide in polysorbate- 80 coated polybutylcyanoacrylate nanoparticles. Int. J. Nanomedicine 6, 445-452 (2011).

6 Jain A, Chasoo G, Singh SK et al. Transferrin-appended PEGylated nanoparticles for temozolomide delivery to brain: in vitro characterisation. J. Microencapsul. 28, 21-28 (2011).

7 Irani M, Mir Mohamad Sadeghi G, Haririan I. A novel biocompatible drug delivery system of chitosan/ temozolomide nanoparticles loaded PCL-PU nanofibers for sustained delivery of temozolomide. Int. J. Biol. Macromol. 97, 744-751 (2017).

8 Fan L, Yang Q, Tan J et al. Dual loading miR-218 mimics and temozolomide using AuCOOH@FA-CS drug delivery system: promising targeted anti-tumor drug delivery system with sequential release functions. J. Exp. Clin. Cancer Res. 34, 106 (2015). is nose-to-brain targeting that could improve the TMZ concentrations in the brain. For a translational nasal delivery system, consideration should be given to the required maximum dose that could be delivered via this route along with formulation properties that improve retention and, hence, permeation through the nasal route.

\section{Financial \& competing interests disclosure}

The authors have no relevant affiliations or financial involvement with any organization or entity with a financial interest in or financial conflict with the subject matter or materials discussed in the manuscript. This includes employment, consultancies, honoraria, stock ownership or options, expert testimony, grants or patents received or pending, or royalties.

No writing assistance was utilized in the production of this manuscript.

Di Martino A, Sedlarik V. Amphiphilic chitosan-graftedfunctionalized polylactic acid based nanoparticles as a delivery system for doxorubicin and temozolomide cotherapy. Int. J. Pharm. 474, 134-145 (2014).

10 Huang G, Zhang N, Bi X et al. Solid lipid nanoparticles of temozolomide: potential reduction of cardiac and nephric toxicity. Int. J. Pharm. 355, 314-320 (2008).

11 Song S, Mao G, Du J et al. Novel RGD containing, temozolomide-loading nanostructured lipid carriers for glioblastoma multiforme chemotherapy. Drug Deliv. 23, 1404-1408 (2016).

12 Wu M, Fan Y, Lv S et al. Vincristine and temozolomide combined chemotherapy for the treatment of glioma: a comparison of solid lipid nanoparticles and nanostructured lipid carriers for dual drugs delivery. Drug Deliv. 23, 2720-2725 (2016).

13 Ananta JS, Paulmurugan R, Massoud TF. Temozolomideloaded PLGA nanoparticles to treat glioblastoma cells: a biophysical and cell culture evaluation. Neurol. Res. 38, 51-59 (2016).

14 Lee CY, Ooi IH. Preparation of temozolomide-loaded nanoparticles for glioblastoma multiforme targeting-ideal versus reality. Pharmaceuticals (Basel) 9, 54 (2016).

15 Patil R, Portilla-Arias J, Ding $\mathrm{H}$ et al. Temozolomide delivery to tumor cells by a multifunctional nano-vehicle based on poly(beta-L-malic acid). Pharm. Res. 27, 2317-2329 (2010).

16 Cho HY, Wang W, Jhaveri N et al. NEO212, temozolomide conjugated to perillyl alcohol, is a novel drug for effective treatment of a broad range of temozolomide-resistant gliomas. Mol. Cancer. Ther. 13, 2004-2017 (2014). 
